\title{
NLO effects for doubly heavy baryons in QCD sum rules
}

\author{
Chen-Yu Wang, ${ }^{1, *}$ Ce Meng, ${ }^{1, \dagger}$ Yan-Qing Ma, ${ }^{1,2,3, *}$ and Kuang-Ta Chao ${ }^{1,2,3, \S}$ \\ ${ }^{1}$ School of Physics and State Key Laboratory of Nuclear Physics and Technology, \\ Peking University, Beijing 100871, China \\ ${ }^{2}$ Center for High Energy Physics, Peking University, Beijing 100871, China \\ ${ }^{3}$ Collaborative Innovation Center of Quantum Matter, Beijing 100871, China
}

(Received 17 August 2017; published 14 January 2019)

\begin{abstract}
With the QCD sum rules approach, we study the newly discovered doubly heavy baryon $\Xi_{c c}^{++}$. We analytically calculate the next-to-leading-order (NLO) contribution to the perturbative part of the $J^{P}=\frac{1}{2}^{+}$baryon current with two identical heavy quarks, and then reanalyze the mass of $\Xi_{c c}^{++}$at the NLO level. We find that the NLO correction significantly improves both scheme dependence and scale dependence, whereas it is hard to control these theoretical uncertainties at leading order. With the NLO contribution, the baryon mass is estimated to be $m_{\Xi_{c c}^{++}}=3.66_{-0.10}^{+0.08} \mathrm{GeV}$, which is consistent with the LHCb measurement.
\end{abstract}

DOI: 10.1103/PhysRevD.99.014018

\section{INTRODUCTION}

The quark model predicts rich structures of hadronic states with various flavors. Numerous predicted states have been observed experimentally, indicating the validity of the quark model classification for hadrons. However, a class of states, which contain more than one heavy quark, have not been discovered for decades. Recently, the LHCb Collaboration observed a highly significant structure in the $\Lambda_{c}^{+} K^{-} \pi^{+} \pi^{+}$ mass spectrum, which is interpreted as the doubly charmed baryon $\Xi_{c c}^{++}[1]$ with mass $3621 \pm 0.72 \pm 0.27 \pm 0.14 \mathrm{MeV}$. Early experimental studies of $\Xi_{c c}^{+}$were performed by the SELEX [2], BABAR [3], and Belle [4] collaborations.

The understanding of $\Xi_{c c}^{++}$demands more rigorous theoretical studies. Plenty of methods have been used in the literature [5-13]. Among them, the QCD sum rules, which are based on the first principle of QCD, are powerful tools to study various properties of hadronic states [14,15]. Many works have been devoted to the study of doubly heavy baryons within QCD sum rules [16-22], and some impressive predictions have been obtained. But in all of these works, only the leading order (LO) in the $\alpha_{s}$ expansion of perturbative contributions and Wilson coefficients of vacuum condensates were considered. Without

\footnotetext{
*wangcy@pku.edu.cn

†mengce75@pku.edu.cn

†yma@pku.edu.cn

${ }^{\S}$ ktchao@pku.edu.cn
}

Published by the American Physical Society under the terms of the Creative Commons Attribution 4.0 International license. Further distribution of this work must maintain attribution to the author(s) and the published article's title, journal citation, and DOI. Funded by SCOAP. higher-order contributions, it is hard to control theoretical uncertainties in QCD sum rules, which limits its predictive power. For instance, at LO the value of the charm-quark mass cannot be well determined, which can cause large errors. In fact, it was known a long time ago that the nextto-leading-order (NLO) correction has sizable contributions to meson and nucleon sum rules [23-25]. Therefore, the study of NLO effects for doubly heavy baryons in QCD sum rules is badly needed.

Higher-order calculations in QCD sum rules become harder and harder when more particles or more massive particles are involved. For mesons, the state-of-the-art calculation has been developed to $\mathcal{O}\left(\alpha_{s}^{4}\right)$ with the help of mass expansion [26-31], while for baryons, the $\mathcal{O}\left(\alpha_{s}\right)$ correction is available in the literature only for nucleons and singly heavy baryons $[24,25,32]$.

In this paper, we calculate the NLO correction to the perturbative contribution for the doubly heavy $J^{P}=\frac{1}{2}+$ baryon, and show its important effects in QCD sum rules. With the help of the integration-by-parts [33,34] and differential equation $[35,36]$ methods, we get a fully analytical expression. We reproduce the massless result in the literature when we set all quark masses to zero. Based on this calculation, we reanalyze the newly discovered $\Xi_{c c}^{++}$in QCD sum rules.

\section{QCD SUM RULES}

The central object in QCD sum rules is the following two-point correlation function [14,37]:

$$
\begin{aligned}
\Pi(q) & =i \int \mathrm{d}^{4} x e^{i q x}\langle\Omega|T\{\eta(x) \bar{\eta}(0)\}| \Omega\rangle \\
& =\Pi_{1}\left(q^{2}\right) \not q+\Pi_{2}\left(q^{2}\right)
\end{aligned}
$$


where $\Omega$ denotes the QCD vacuum and $\eta$ is the baryon current (to be defined later).

On the one hand, one can calculate $\Pi(q)$ using the operator product expansion (OPE), which gives

$$
\Pi(q)=C_{1}(q)+\sum_{i} C_{i}(q)\left\langle O_{i}\right\rangle,
$$

where $C_{1}$ is the perturbative contribution and $C_{i}$ is the Wilson coefficient of a gauge-invariant Lorentz scalar operator $O_{i}$. Both $C_{1}$ and $C_{i}$ are perturbatively calculable. $\left\langle O_{i}\right\rangle$ is a shorthand for the vacuum condensates $\left\langle\Omega\left|O_{i}\right| \Omega\right\rangle$, which is a nonperturbative but universal quantity. It means that the value of $\left\langle O_{i}\right\rangle$ determined from other processes should be the same as its value in the process considered in this paper.

On the other hand, $\Pi(q)$ satisfies the dispersion relation

$$
\begin{aligned}
\Pi(q) & =\frac{1}{\pi} \int_{0}^{\infty} \mathrm{d} s \frac{\Im \Pi_{1}(s+i \epsilon) \not 1+\Im \Pi_{2}(s+i \epsilon)}{s-q^{2}} \\
& =\int_{0}^{\infty} \mathrm{d} s \frac{\rho_{1}(s+i \epsilon) \not 1+\rho_{2}(s+i \epsilon)}{s-q^{2}},
\end{aligned}
$$

where $\rho_{1}$ and $\rho_{2}$ are the spectrum densities. Based on the optical theorem, one assumes the spectrum density $\rho(q)=\rho_{1}\left(q^{2}\right) q q+\rho_{2}\left(q^{2}\right)$ to be [37]

$\rho(q)=\lambda_{H}^{2}\left(\not q+m_{H}\right) \delta\left(q^{2}-m_{H}^{2}\right)+\rho_{c}(q) \theta\left(q^{2}-s_{\mathrm{th}}\right)$,

where $s_{\mathrm{th}}$ is the threshold of the continuum spectrum and $\lambda_{H}$ is defined by $\lambda_{H} u(p, s)=\langle 0|\eta(0)| H(p, s)\rangle$, where $u(p, s)$ is the Dirac spinor of the hadron.

By defining

$$
\begin{aligned}
& \frac{\Im C_{1}(q)}{\pi}=\rho_{1,1}\left(q^{2}\right) \not q+\rho_{2,1}\left(q^{2}\right), \\
& \frac{\Im C_{i}(q)}{\pi}=\rho_{1, i}\left(q^{2}\right) \not 1+\rho_{2, i}\left(q^{2}\right),
\end{aligned}
$$

and employing the quark-hadron duality and Borel transformation, we obtain a sum rule corresponding to $\Pi_{1}\left(q^{2}\right)[37]$,

$$
\begin{aligned}
\lambda_{H}^{2} e^{-\frac{m_{H}^{2}}{m_{B}^{2}}}= & \int_{s_{\mathrm{th}}}^{s_{0}} \mathrm{~d} s \rho_{1,1}(s) e^{-\frac{s}{m_{B}^{2}}} \\
& +\sum_{i}\left\langle O_{i}\right\rangle \int_{s_{\mathrm{th}}}^{\infty} \mathrm{d} s \rho_{1, i}(s) e^{-\frac{s}{m_{B}^{2}}},
\end{aligned}
$$

where $s_{0}$ is the threshold parameter and $m_{B}$ is the Borel parameter, which are introduced in the quark-hadron duality and Borel transformation, respectively. One can also obtain a similar sum rule corresponding to $\Pi_{2}\left(q^{2}\right)$, but we will not discuss it in this paper.

To obtain the baryon mass, we differentiate both sides of Eq. (7) with respect to $-m_{B}^{-2}$ and solve for $m_{H}^{2}$, which results in
$m_{H}^{2}=\frac{\int_{s_{\mathrm{th}}}^{s_{0}} \mathrm{~d} s \rho_{1,1}(s) s e^{-\frac{s}{m_{B}^{2}}}+\sum_{i}\left\langle O_{i}\right\rangle \int_{s_{\mathrm{th}}}^{\infty} \mathrm{d} s \rho_{1, i}(s) s e^{-\frac{s}{m_{B}^{2}}}}{\int_{s_{\mathrm{th}}}^{s_{0}} \mathrm{~d} s \rho_{1,1}(s) e^{-\frac{s}{m_{B}^{2}}}+\sum_{i}\left\langle O_{i}\right\rangle \int_{s_{\mathrm{th}}}^{\infty} \mathrm{d} s \rho_{1, i}(s) e^{-\frac{s}{m_{B}^{2}}}}$.

In this paper, as a good approximation we only keep vacuum condensates up to dimension four,

$$
\left\langle O_{i}\right\rangle \in\left\{\left\langle\bar{q}_{j}^{a} q_{j}^{a}\right\rangle,\left\langle g_{s}^{2} G_{\mu \nu}^{a} G^{a \mu \nu}\right\rangle\right\},
$$

and evaluate $\rho_{1,\langle\bar{q} q\rangle}$ up to $\mathcal{O}\left(m_{q}\right)$. Contributions of higherdimensional operators are power suppressed and thus can be neglected. (See Appendix B for more discussions on higher-dimensional operators.)

\section{BARYON CURRENTS}

The most general baryon current containing two identical heavy quarks is

$$
\epsilon^{a b c}\left(Q^{a} C \Gamma_{1} Q^{b}\right) \Gamma_{2} q^{c},
$$

where $Q$ is the heavy quark with mass $m_{Q}$ and $q$ is the light quark with mass $m_{q} . \epsilon^{a b c}$ is the antisymmetric matrix in color space, $C$ is the charge-conjugation matrix, and $\Gamma_{1}$ and $\Gamma_{2}$ are Dirac matrices with possible Lorentz indices suppressed. Spinor indices are contracted within the bracket, and therefore transposing the bracket part should keep the current intact. Noting that $C^{T}=-C$, one can see that $\Gamma_{1}$ can only be $\gamma_{\mu}$ or $\sigma_{\mu \nu}$ [37]. For a $J^{P}=\frac{1}{2}+$ baryon, there are only two possible currents:

$$
\begin{gathered}
\eta_{1}=\epsilon^{a b c}\left(Q^{a} C \gamma_{\mu} Q^{b}\right) \gamma^{\mu} \gamma^{5} q^{c}, \\
\eta_{2}=\epsilon^{a b c}\left(Q^{a} C \sigma_{\mu \nu} Q^{b}\right) \sigma^{\mu \nu} i \gamma^{5} q^{c},
\end{gathered}
$$

where $\eta_{1}$ corresponds to the Ioffe current [37] if we take $Q$ as a $u$ quark and $q$ as a $d$ quark. It is well known that $\eta_{1}$ and $\eta_{2}$ are renormalization covariant [38],

$$
\frac{\mathrm{d}}{\mathrm{d} \ln \mu^{2}}\left(\begin{array}{l}
\eta_{1} \\
\eta_{2}
\end{array}\right)=\left(\begin{array}{cc}
\gamma_{1} & 0 \\
0 & \gamma_{2}
\end{array}\right)\left(\begin{array}{l}
\eta_{1} \\
\eta_{2}
\end{array}\right)
$$

Thus, it is advantageous to work with these currents when calculating the NLO correction. There exist other choices for the current $[16,39,40]$, which can be expressed by $\eta_{1}$ and $\eta_{2}$ with the help of the Fierz identity,

$$
\begin{aligned}
\eta_{\text {mix }} & =\epsilon^{a b c}\left[\left(Q^{a} C \gamma^{5} q^{b}\right) Q^{c}+b\left(Q^{a} C q^{b}\right) \gamma^{5} Q^{c}\right] \\
& =\frac{b-1}{4} \eta_{1}+i \frac{b+1}{8} \eta_{2},
\end{aligned}
$$

where $b$ is a complex mixing parameter. 


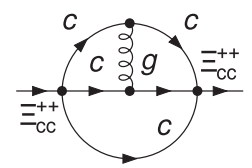

$u$

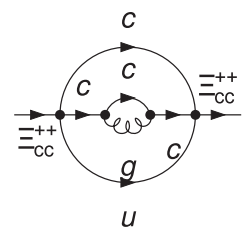

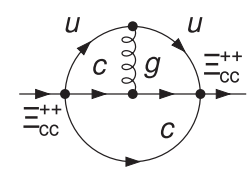

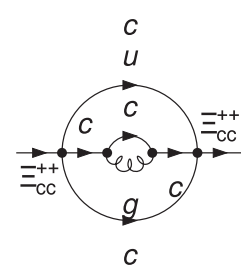

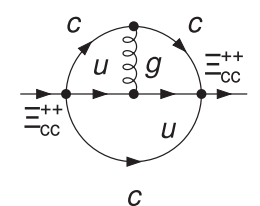

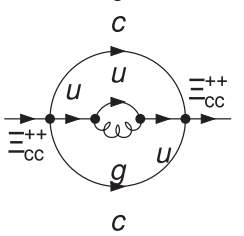

FIG. 1. NLO Feynman diagrams for $C_{1}$. External legs are amputated.

\section{NLO CORRECTION TO $C_{1}$}

It is known that $C_{1}$ and $C_{i}$ can be calculated perturbatively, and results at LO are available in Refs. [16,41]. Among them, the most important one is $C_{1}$, because all other coefficients will be multiplied by higher-dimensional operators which are power suppressed. Thus the main theoretical uncertainty is due to the NLO correction to $C_{1}$.

In order to perform the NLO calculation for $C_{1}$, we use FEYNARTs $[42,43]$ to generate all Feynman diagrams (see Fig. 1) and FeYNCALC [44,45] to manipulate the resulting amplitude. After these steps, we are left with some threeloop-like scalar integrals. These integrals can be further simplified using the integration-by-parts (IBP) method [33,34]. FIRE [46] and LITERED [47] are used to reduce the full amplitude to a linear combination of a complete set of 29 master integrals (see Fig. 2),

$$
C_{1}^{\mathrm{NLO}}\left(\varepsilon, q, m_{Q}\right)=\sum_{k} c_{k}\left(\varepsilon, q, m_{Q}\right) I_{k}(\varepsilon, v),
$$

where $\varepsilon$ is defined by the dimension $D=4-2 \varepsilon$, $v=\sqrt{1-\frac{4 m_{Q}^{2}}{q^{2}}}$, and all coefficients $c_{k}$ are purely imaginary. Note that here $I_{k}$ is defined to be dimensionless.

Since we are only interested in the imaginary part of the two-point function $\Pi\left(p^{2}\right)$, we just need to evaluate the corresponding cut diagrams of $I_{k}$. But evaluating these four-body phase-space integrals in the presence of two massive particles is still a formidable task.

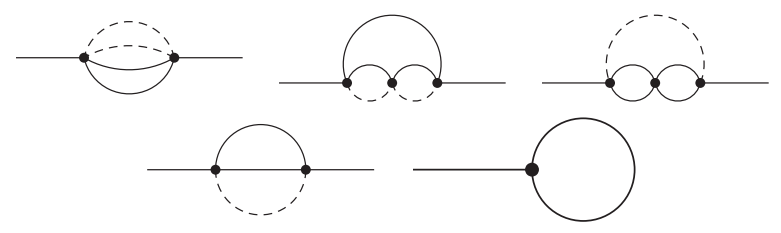

FIG. 2. Topologies of master integrals, where solid and dashed lines denote massive and massless propagators, respectively. External legs are amputated.
To proceed, we employ the differential equation method $[35,36]$ by first differentiating $I_{k}$ with respect to $v$, then reducing the resulting integrals by using IBP, and obtaining a system of differential equations,

$$
\frac{\mathrm{d} \boldsymbol{I}(\varepsilon, v)}{\mathrm{d} v}=\boldsymbol{A}(\varepsilon, v) \boldsymbol{I}(\varepsilon, v),
$$

where $\boldsymbol{I}$ represents the vector of master integrals $I_{k}$ and $\boldsymbol{A}$ is a $29 \times 29$ matrix. To solve this differential equation, we implement the algorithm proposed in Ref. [48] to transform the equation into the so-called $\varepsilon$ form [35],

$$
\frac{\mathrm{d} \boldsymbol{I}^{\prime}(\varepsilon, v)}{\mathrm{d} v}=\varepsilon \sum_{i} \frac{\boldsymbol{B}_{i}}{v-v_{i}} \boldsymbol{I}^{\prime}(\varepsilon, v)
$$

where $v_{i} \in\{0, \pm 1, \pm \sqrt{3} i\}, \boldsymbol{B}_{i}$ are constant matrices, and $\boldsymbol{I}^{\prime}$ is related to $\boldsymbol{I}$ by an invertible linear transformation. The virtue of this $\varepsilon$ form is that the right-hand side of Eq. (17) is proportional to $\varepsilon$, which can be easily solved iteratively in terms of Goncharov polylogarithms [49]. The boundary values of $\boldsymbol{I}(\varepsilon, v)$ at $v=1$, i.e., $m_{Q}=0$, are nothing but massless four-body phase-space integrals, which are very easy to work out. By evaluating the boundary value $\boldsymbol{I}(\varepsilon, 1)$ and solving the equation iteratively, we finally obtain $I_{k}$ and finish our calculation.

We find that the Coulombic singularity, which may appear as $v \rightarrow 0$, does not present at NLO. Then, by combining all of the terms the infrared divergences are canceled out, so we only need to deal with ultraviolet divergences. After performing wave-function and mass renormalization of quarks $\left(m_{Q}\right.$ is renormalized in either the $\overline{\mathrm{MS}}$ scheme or on-shell scheme), the remaining ultraviolet divergences can be removed by operator renormalization of $\eta_{1}$ and $\eta_{2}$. We renormalize them in the $\overline{\mathrm{MS}}$ scheme, of which the anomalous dimensions are

$$
\gamma_{1}=\gamma_{2}=\frac{\alpha_{s}}{2 \pi},
$$

which confirms the results in Refs. $[25,50]$.

We then get a finite result at NLO. Our NLO result confirms the massless result $[24,25]$ in the limit $m_{Q} \rightarrow 0$. Our analytical result is listed in Appendix A.

\section{PHENOMENOLOGY}

In our analysis we use

$$
\eta=\eta_{1}+\theta \eta_{2}
$$

where $\theta$ is a complex mixing parameter. We choose the following parameters [16,51-54]:

$$
\begin{aligned}
& m_{u}(2 \mathrm{GeV})=2.36 \pm 0.24 \mathrm{MeV}, \\
& m_{d}(2 \mathrm{GeV})=5.03 \pm 0.26 \mathrm{MeV},
\end{aligned}
$$



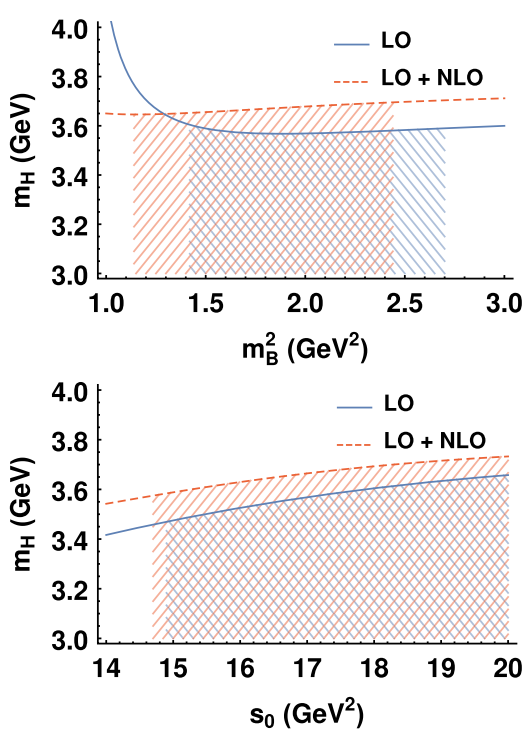

FIG. 3. Prediction of $m_{\Xi_{c c}^{++}}$as a function of $m_{B}^{2}$ and $s_{0}$. Shadows correspond to the windows defined by Eq. (28).

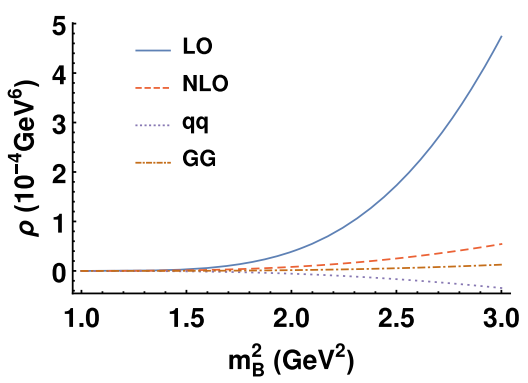

FIG. 4. Contributions of various terms on the right-hand side of Eq. (7).

$$
\begin{gathered}
m_{c}^{\overline{\mathrm{MS}}}\left(m_{c}\right)=1.28 \pm 0.03 \mathrm{GeV}, \\
m_{c}^{\text {on-shell }}=1.46 \pm 0.07 \mathrm{GeV}, \\
\langle\bar{q} q\rangle(2 \mathrm{GeV})=-(0.280 \pm 0.017 \mathrm{GeV})^{3}, \\
\left\langle g_{s}^{2} G G\right\rangle=4 \pi^{2}(0.037 \pm 0.015) \mathrm{GeV}^{4},
\end{gathered}
$$

and $\alpha_{s}\left(m_{Z}=91.1876 \mathrm{GeV}\right)=0.1181$. The $m_{c}^{\text {on-shell }}$ comes from the QCD sum rules analysis of the $J / \psi$ spectrum, in which the mass renormalization scheme and the truncation order of $\alpha_{s}$ of $C_{1}$ are the same as ours. Thus, it is consistent to use this on-shell quark mass in our analysis. According to Eq. (8), the evolution of the current $\eta$ is irrelevant to the estimation of the hadron mass, and thus we do not include it in our analysis. We use the two-loop running for the coupling constant $\alpha_{s}$ and heavy quark mass $m_{Q}$. The vacuum condensates are evolved according to their oneloop anomalous dimensions: $\gamma_{\langle\bar{q} q\rangle}=-\gamma_{m_{q}}$ and $\gamma_{\left\langle g_{s}^{2} G G\right\rangle}=0$ [55]. In the following, unless otherwise stated, we choose central values for all parameters, set the renormalization scale $\mu=m_{B}[14,56]$, and choose the $\overline{\mathrm{MS}}$ scheme for the heavy quark mass renormalization.

In Eq. (8), the baryon mass $m_{H}$ depends on two parameters: $m_{B}$ and $s_{0}$. In order to obtain a reliable result, we should keep $m_{B}$ inside the so-called Borel window to ensure the validity of the OPE, and the choice of $s_{0}$ should ensure that the groundstate pole contribution is dominant. Since $m_{H}$ is a property of the hadron, it does not depend on $m_{B}$ and $s_{0}$, and thus within the valid parameter space (we shall call it the "window" hereafter) we should find the region in which $m_{H}$ depends weakly on $m_{B}$ and $s_{0} . m_{H}$ in this region is considered to be the estimated hadron mass in QCD sum rules.

We define the relative contributions of the condensates and continuum spectrum as

$$
\begin{gathered}
r_{i}=\frac{\left\langle O_{i}\right\rangle \int_{s_{\mathrm{th}}}^{\infty} \mathrm{d} s \rho_{1, i}(s) e^{-\frac{s}{m_{B}^{2}}}}{\int_{s_{\mathrm{th}}}^{\infty} \mathrm{d} s \rho_{1,1}(s) e^{-\frac{s}{m_{B}^{2}}}}, \\
r_{\text {cont }}=\frac{\int_{s_{0}}^{\infty} \mathrm{d} s \rho_{1,1}(s) e^{-\frac{s}{m_{B}^{2}}}}{\int_{s_{\mathrm{th}}}^{\infty} \mathrm{d} s \rho_{1,1}(s) e^{-\frac{s}{m_{B}^{2}}}},
\end{gathered}
$$

and impose the following constraints on our sum rule:

$$
\left|r_{i}\right| \leq 30 \%, \quad\left|\sum_{i} r_{i}\right| \leq 30 \%, \quad\left|r_{\text {cont }}\right| \leq 30 \% .
$$

We find that with a mixing parameter $\theta=0.018 i$, we can obtain very stable plateaux for $m_{B}$ and $s_{0}$, as shown in

TABLE I. Parameters for plateaux and predictions for $m_{\Xi_{c c}^{++}}$in different mixing and mass renormalization schemes.

\begin{tabular}{lclcccccc}
\hline \hline$\theta$ & $m_{Q}$ scheme & Order & $m_{B}^{2}\left(\mathrm{GeV}^{2}\right)$ & $s_{0}\left(\mathrm{GeV}^{2}\right)$ & $m_{\Xi_{c c}^{++}}(\mathrm{GeV})$ & Error from $m_{B}^{2}$ & Error from $s_{0}$ & Error from $m_{Q}$ \\
\hline $0.018 i$ & $\overline{M S}$ & LO & $2.0 \pm 0.3$ & $17 \pm 2$ & $3.57_{-0.11}^{+0.08}$ & $-0.00+0.01$ & $-0.09+0.07$ & $-0.05+0.05$ \\
& & NLO & $1.7 \pm 0.3$ & $17 \pm 2$ & $3.66_{-0.10}^{+0.08}$ & $-0.01+0.01$ & $-0.08+0.05$ & $-0.05+0.05$ \\
\multirow{2}{*}{$0.018 i$} & on-shell & LO & $1.7 \pm 0.3$ & $17 \pm 2$ & $3.83_{-0.14}^{+0.13}$ & $-0.03+0.00$ & $-0.09+0.07$ & $-0.10+0.10$ \\
& & NLO & $1.4 \pm 0.3$ & $17 \pm 2$ & $3.65_{-0.14}^{+0.11}$ & $-0.07+0.05$ & $-0.08+0.05$ & $-0.10+0.09$ \\
$-\frac{i}{3}$ & $\overline{M S}$ & LO & $4.4 \pm 0.3$ & $23 \pm 2$ & $3.81_{-0.11}^{+0.10}$ & $-0.04+0.04$ & $-0.10+0.08$ & $-0.03+0.03$ \\
& & NLO & $4.0 \pm 0.3$ & $23 \pm 2$ & $3.86_{-0.11}^{+0.10}$ & $-0.05+0.04$ & $-0.09+0.08$ & $-0.03+0.03$ \\
\hline \hline
\end{tabular}




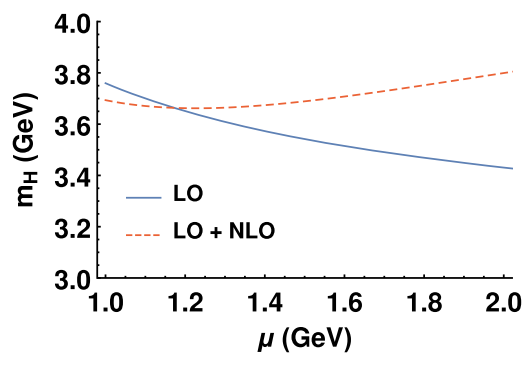

FIG. 5. Prediction of $m_{\Xi_{c c}^{++}}$as a function of $\mu$.

Fig. 3. Note, however, that QCD sum rules alone cannot tell which mixing current is the most suitable for a QCD sum rules analysis. For example, there is a family of mixing parameters that can yield similar good plateaux for $m_{B}$ and $s_{0}$, and a similar estimation of $m_{H}$. We also provide another set of results by choosing $\theta=-\frac{i}{3}$, which corresponds to the mixing used in Ref. [16].

The relative importance of each term in the OPE is shown in Fig. 4, where $m_{B}^{2}$ and $s_{0}$ are set to their central values shown in Table I. We find that the NLO correction has an important contribution. In the $m_{Q}^{\overline{\mathrm{MS}}}$ scheme the ratio of the NLO correction to LO is about 29\% (19\%) for $\theta=0.018 i\left(\theta=-\frac{i}{3}\right)$, while in the $m_{Q}^{\text {on-shell }}$ scheme this ratio reaches $233 \%$ for $\theta=0.018 i$, signaling the bad convergence of the perturbative expansion, which is the reason why we chose the $\overline{\mathrm{MS}}$ scheme by default. Nevertheless, with the NLO correction, the difference between the predicted $m_{\Xi_{c c}^{++}}$in the $\overline{\mathrm{MS}}$ scheme and that in the on-shell scheme for $m_{Q}$ is substantially reduced. As shown in Table I, the mass differences obtained from the LO and $\mathrm{LO}+\mathrm{NLO}$ results are 0.27 and $0.01 \mathrm{GeV}$, respectively. Thus, the NLO correction largely reduces the scheme dependence.

To study the renormalization scale $\mu$ dependence, we fix all other parameters to their default choices (or central values) and freely vary $\mu$. The variation of $m_{\Xi_{c c}^{++}}$with respect to $\mu$ is shown in Fig. 5. We find that the scale dependence is much weaker when the NLO correction is included. More precisely, the error of $m_{\Xi_{c c}^{++}}$induced by $\mu=m_{B} \pm 0.2 \mathrm{GeV}$ is ${ }_{-0.08}^{+0.06} \mathrm{GeV}$ and ${ }_{-0.01}^{+0.03} \mathrm{GeV}$ for $\mathrm{LO}$ and $\mathrm{LO}+\mathrm{NLO}$, respectively.
Our final results for $m_{\Xi_{c c}^{++}}$are shown in Table I. The errors of $m_{B}^{2}, s_{0}$, and the parameters listed in Eqs. (20)-(25) are used to determine the error of $m_{\Xi_{c c}^{+}}$. We find that our NLO result is consistent with the LHCb measurement. As a comparison, we also list the results from the $m_{Q}^{\text {on-shell }}$ renormalization scheme or with $\theta=-\frac{i}{3}$. We find that all of the plots above are almost unchanged when changing $m_{q}$ from $m_{u}$ to $m_{d}$, and thus our prediction of the mass of $\Xi_{c c}^{+}(c c d)$ is almost the same as that of $\Xi_{c c}^{++}(c c u)$.

\section{SUMMARY}

The NLO calculation for hadrons with massive quarks in QCD sum rules is important but hard to carry out. With the help of the recent development of multiloop calculation techniques, we are able to analytically calculate the NLO perturbative correction to the imaginary part of the two-point correlation function of the $J^{P}=\frac{1}{2}+$ baryon current with two identical heavy quarks. We apply our result to the QCD sum rules analysis of the newly discovered baryon $\Xi_{c c}^{++}$by the LHCb Collaboration [1]. The QCD sum rules estimation of $m_{\Xi_{c c}^{++}}$is $3.66_{-0.10}^{+0.08} \mathrm{GeV}$, which is consistent with the $\mathrm{LHCb}$ measurement within uncertainties. By comparing $\mathrm{LO}$ with $\mathrm{LO}+\mathrm{NLO}$ results, we find that the NLO perturbative correction substantially reduces the $m_{Q}$ renormalization scheme dependence and renormalization scale $\mu$ dependence, and thus brings the theoretical uncertainties under better control.

\section{ACKNOWLEDGMENTS}

We thank H.X. Chen and S. L. Zhu for many useful communications and discussions. This work is supported in part by the National Natural Science Foundation of China (Grants No. 11475005 and No. 11075002) and the National Key Basic Research Program of China (No. 2015CB856700), and the High-performance Computing Platform of Peking University.

\section{APPENDIX A: ANALYTICAL RESULT}

We calculate various spectrum densities of the current defined in Eq. (19). The corresponding LO spectrum densities, defined in Eq. (5), are

$$
\begin{aligned}
\rho_{1,1}^{\mathrm{LO}}= & \frac{1}{2048 \pi^{4}} q^{4}\left[2 v\left(9 v^{6}-9 v^{4}+31 v^{2}-15\right)+3\left(v^{2}-1\right)^{3}\left(3 v^{2}+5\right) \ln \left(\frac{1-v}{1+v}\right)\right] \\
& +\frac{3}{1024 \pi^{4}} q^{4}|\theta|^{2}\left[2 v\left(3 v^{6}-11 v^{4}+69 v^{2}-45\right)+3\left(v^{2}-1\right)^{2}\left(v^{4}-2 v^{2}-15\right) \ln \left(\frac{1-v}{1+v}\right)\right], \\
& \rho_{2,1}^{\mathrm{LO}}=\frac{3}{128 \pi^{4}} q^{4} m_{Q} \Im \theta\left[2 v\left(v^{2}+3\right)\left(3 v^{2}-5\right)+3\left(v^{2}-1\right)\left(v^{4}+2 v^{2}+5\right) \ln \left(\frac{1-v}{1+v}\right)\right],
\end{aligned}
$$




$$
\begin{gathered}
\rho_{1,\langle\bar{q} q\rangle}^{\mathrm{LO}}=\frac{3}{\pi^{2}} m_{Q} \Im \theta v-\frac{1}{32 \pi^{2}} m_{q} \frac{v^{4}-6 v^{2}-3}{v}-\frac{3}{8 \pi^{2}} m_{q}|\theta|^{2} \frac{v^{4}-4 v^{2}-1}{v}, \\
\rho_{2,\langle\bar{q} q\rangle}^{\mathrm{LO}}=\frac{1}{8 \pi^{2}} q^{2} v\left(v^{2}-3\right)+\frac{3}{2 \pi^{2}} q^{2}|\theta|^{2} v\left(v^{2}-1\right)-\frac{3}{4 \pi^{2}} m_{Q} m_{q} \Im \theta \frac{3 v^{2}+1}{v}, \\
\rho_{1,\left\langle g_{s}^{2} G G\right\rangle}^{\mathrm{LO}}=\frac{1}{512 \pi^{4}}\left[2 v\left(v^{2}+1\right)+\left(v^{2}-1\right)^{2} \ln \left(\frac{1-v}{1+v}\right)\right]-\frac{1}{256 \pi^{4}}|\theta|^{2}\left[2 v\left(v^{2}+1\right)+\left(v^{2}-1\right)^{2} \ln \left(\frac{1-v}{1+v}\right)\right], \\
\rho_{2,\left\langle g_{s}^{2} G G\right\rangle}^{\mathrm{LO}}=-\frac{1}{64 \pi^{4}} m_{Q} \Im \theta\left[\frac{2 v\left(3 v^{2}-11\right)}{v^{2}-1}+\left(v^{2}+11\right) \ln \left(\frac{1-v}{1+v}\right)\right] .
\end{gathered}
$$

With the help of Eq. (14), our result confirms previous calculations [16,41].

The NLO spectrum densities of the perturbative contribution in the $\overline{\mathrm{MS}}$ scheme, with $m_{Q}$ also renormalized in the $\overline{\mathrm{MS}}$ scheme, are

$$
\begin{aligned}
& \left.\rho_{1,1}^{\mathrm{NLO}}\right|_{m_{Q}^{\overline{\mathrm{MS}}}}=\frac{\alpha_{s}}{2 \pi}\left[2 \rho_{1,1}^{\mathrm{LO}} \ln \left(\frac{\mu^{2}}{q^{2}}\right)+2 \rho_{a}^{m_{Q}} \ln \left(\frac{\mu^{2}}{q^{2}}\right)+\rho_{a}\right], \\
& \left.\rho_{2,1}^{\mathrm{NLO}}\right|_{m_{Q}^{\overline{\mathrm{MS}}}}=\frac{\alpha_{s}}{2 \pi}\left[2 \rho_{2,1}^{\mathrm{LO}} \ln \left(\frac{\mu^{2}}{q^{2}}\right)+2 \rho_{b}^{m_{Q}} \ln \left(\frac{\mu^{2}}{q^{2}}\right)+\rho_{b}\right],
\end{aligned}
$$

where $\rho_{a}^{m_{Q}}$ and $\rho_{b}^{m_{Q}}$ come from $m_{Q}$ renormalization,

$$
\rho_{a}^{m_{Q}}=m_{Q} \frac{\partial}{\partial m_{Q}} \rho_{1,1}^{\mathrm{LO}}, \quad \rho_{b}^{m_{Q}}=m_{Q} \frac{\partial}{\partial m_{Q}} \rho_{2,1}^{\mathrm{LO}} .
$$

The analytical expressions of $\rho_{a}$ and $\rho_{b}$ will be presented later. The differences between the $m_{Q}^{\text {on-shell }}$ scheme and $m_{Q}^{\overline{\mathrm{MS}}}$ scheme are

$$
\begin{aligned}
& \left.\rho_{1,1}^{\mathrm{NLO}}\right|_{m_{Q}^{\text {on-shell }}}=\left.\rho_{1,1}^{\mathrm{NLO}}\right|_{m_{Q}^{\overline{\mathrm{MS}}}}-\frac{\alpha_{s}}{2 \pi}\left[\frac{8}{3}+2 \ln \left(\frac{\mu^{2}}{m_{Q}^{2}}\right)\right] \rho_{a}^{m_{Q}}, \\
& \left.\rho_{2,1}^{\mathrm{NLO}}\right|_{m_{Q}^{\text {on-shell }}}=\left.\rho_{2,1}^{\mathrm{NLO}}\right|_{m_{Q}^{\overline{\mathrm{MS}}}}-\frac{\alpha_{s}}{2 \pi}\left[\frac{8}{3}+2 \ln \left(\frac{\mu^{2}}{m_{Q}^{2}}\right)\right] \rho_{b}^{m_{Q}} .
\end{aligned}
$$

Note that in the $m_{Q}^{\text {on-shell }}$ scheme, the logarithms coming from $m_{Q}$ renormalization are completely canceled out; only the logarithms proportional to $\rho^{\mathrm{LO}}$ remain, which come from the quark wave-function renormalization and baryon operator renormalization. Equations (A10) and (A11) are just the consequences of changing the renormalization scheme. To show this explicitly, we first replace all $m_{Q}^{\text {on-shell }}$ by $m_{Q}^{\overline{\mathrm{MS}}}$ in $\rho^{\mathrm{LO}}$ and $\rho^{\mathrm{NLO}}$ in the $m_{Q}^{\text {on-shell }}$ scheme,

$$
m_{Q}^{\text {on-shell }}=m_{Q}^{\overline{\mathrm{MS}}}\left(1+\frac{\alpha_{s}}{2 \pi}\left[\frac{8}{3}+2 \ln \left(\frac{\mu^{2}}{\left(m_{Q}^{\overline{\mathrm{MS}}}\right)^{2}}\right)\right]+\mathcal{O}\left(\alpha_{s}^{2}\right)\right)
$$

Then, we expand $\rho^{\mathrm{LO}}$ and $\rho^{\mathrm{NLO}}$ up to $\mathcal{O}\left(\alpha_{s}\right)$. We take $\rho_{1,1}$ as an example. For $\rho_{1,1}^{\mathrm{LO}}$ we have 


$$
\rho_{1,1}^{\mathrm{LO}}\left(m_{Q}^{\mathrm{on}-\text { shell }}\right)=\rho_{1,1}^{\mathrm{LO}}\left(m_{Q}^{\overline{\mathrm{MS}}}\right)+\frac{\alpha_{s}}{2 \pi}\left[\frac{8}{3}+2 \ln \left(\frac{\mu^{2}}{\left(m_{Q}^{\overline{\mathrm{MS}}}\right)^{2}}\right)\right] \rho_{a}^{m_{Q}}\left(m_{Q}^{\overline{\mathrm{MS}}}\right)+\mathcal{O}\left(\alpha_{s}^{2}\right)
$$

and for $\rho_{1,1}^{\mathrm{NLO}}$ we have

$$
\left.\rho_{1,1}^{\mathrm{NLO}}\right|_{m_{Q}^{\text {on-shell }}}\left(m_{Q}^{\text {on-shell }}\right)=\left.\rho_{1,1}^{\mathrm{NLO}}\right|_{m_{Q}^{\text {on-shell }}}\left(m_{Q}^{\overline{\mathrm{MS}}}\right)+\mathcal{O}\left(\alpha_{s}^{2}\right)
$$

Combining them, we obtain

$$
\begin{aligned}
\rho_{1,1}^{\mathrm{LO}}\left(m_{Q}^{\text {on-shell }}\right)+\left.\rho_{1,1}^{\mathrm{NLO}}\right|_{m_{Q}^{\text {on-shell }}}\left(m_{Q}^{\text {on-shell }}\right)= & \rho_{1,1}^{\mathrm{LO}}\left(m_{Q}^{\overline{\mathrm{MS}}}\right)+\left.\rho_{1,1}^{\mathrm{NLO}}\right|_{m_{Q}^{\text {on-shell }}}\left(m_{Q}^{\overline{\mathrm{MS}}}\right)+\frac{\alpha_{s}}{2 \pi}\left[\frac{8}{3}+2 \ln \left(\frac{\mu^{2}}{\left(m_{Q}^{\overline{\mathrm{MS}}}\right)^{2}}\right)\right] \rho_{a}^{m_{Q}}\left(m_{Q}^{\overline{\mathrm{MS}}}\right) \\
& +\mathcal{O}\left(\alpha_{s}^{2}(\mu)\right)
\end{aligned}
$$

Since the renormalized amplitude should not depend on the renormalization scheme, we thus obtain Eq. (A10). For $\rho_{2,1}$ the result is similar: all we need to do is substitute $\rho_{1,1}^{\mathrm{LO}}$, $\rho_{1,1}^{\mathrm{NLO}}$, and $\rho_{a}^{m_{Q}}$ in the above expressions by $\rho_{2,1}^{\mathrm{LO}}, \rho_{2,1}^{\mathrm{NLO}}$, and $\rho_{b}^{m_{Q}}$, respectively.

As a check, we can verify that in the $m_{Q}^{\overline{\mathrm{MS}}}$ scheme the $\mu$ dependence of $m_{Q}$ in $\rho^{\mathrm{LO}}$ is canceled by the corresponding logarithms in $\rho^{\mathrm{NLO}}$ to $\mathcal{O}\left(\alpha_{s}\right)$. To show this explicitly, we first replace all $m_{Q}(\mu)$ by $m_{Q}(\lambda)$ in $\rho^{\mathrm{LO}}$ and $\rho^{\mathrm{NLO}}$ in the $m_{Q}^{\overline{\mathrm{MS}}}$ scheme,

$m_{Q}(\mu)=m_{Q}(\lambda)\left(1-\frac{\alpha_{s}(\mu)}{\pi} \ln \left(\frac{\mu^{2}}{\lambda^{2}}\right)+\mathcal{O}\left(\alpha_{s}^{2}(\mu)\right)\right)$, where $\lambda$ is another scale that differs from $\mu$. Then we expand $\rho^{\mathrm{LO}}$ and $\rho^{\mathrm{NLO}}$ up to $\mathcal{O}\left(\alpha_{s}(\mu)\right)$, and the $\mu$ dependence of $m_{Q}$ should cancel out up to $\mathcal{O}\left(\alpha_{s}(\mu)\right)$. We take $\rho_{1,1}$ as an example. For $\rho_{1,1}^{\mathrm{LO}}$ we have

$$
\begin{aligned}
\rho_{1,1}^{\mathrm{LO}}\left(m_{Q}(\mu)\right)= & \rho_{1,1}^{\mathrm{LO}}\left(m_{Q}(\lambda)\right)-\frac{\alpha_{s}(\mu)}{\pi} \ln \left(\frac{\mu^{2}}{\lambda^{2}}\right) \rho_{a}^{m_{Q}}\left(m_{Q}(\lambda)\right) \\
& +\mathcal{O}\left(\alpha_{s}^{2}(\mu)\right),
\end{aligned}
$$

and for $\rho_{1,1}^{\mathrm{NLO}}$ we have

$$
\begin{aligned}
\rho_{1,1}^{\mathrm{NLO}}\left(\mu, \alpha_{s}(\mu), m_{Q}(\mu)\right)= & \rho_{1,1}^{\mathrm{NLO}}\left(\mu, \alpha_{s}(\mu), m_{Q}(\lambda)\right) \\
& +\mathcal{O}\left(\alpha_{s}^{2}(\mu)\right) .
\end{aligned}
$$

Combining them, we obtain

$$
\begin{aligned}
\rho_{1,1}^{\mathrm{LO}}\left(m_{Q}(\mu)\right)+\rho_{1,1}^{\mathrm{NLO}}\left(\mu, \alpha_{s}(\mu), m_{Q}(\mu)\right)= & \rho_{1,1}^{\mathrm{LO}}\left(m_{Q}(\lambda)\right)+\frac{\alpha_{s}(\mu)}{2 \pi}\left[2 \rho_{1,1}^{\mathrm{LO}}\left(m_{Q}(\lambda)\right) \ln \left(\frac{\mu^{2}}{q^{2}}\right)+\rho_{a}\left(m_{Q}(\lambda)\right)\right] \\
& +\frac{\alpha_{s}(\mu)}{2 \pi}\left[2 \rho_{a}^{m_{Q}}\left(m_{Q}(\lambda)\right) \ln \left(\frac{\lambda^{2}}{q^{2}}\right)\right]+\mathcal{O}\left(\alpha_{s}^{2}(\mu)\right)
\end{aligned}
$$

For $\rho_{2,1}$ the result is similar: all we need to do is substitute $\rho_{1,1}^{\mathrm{LO}}, \rho_{1,1}^{\mathrm{NLO}}, \rho_{a}$, and $\rho_{a}^{m_{Q}}$ in the above expressions by $\rho_{2,1}^{\mathrm{LO}}, \rho_{2,1}^{\mathrm{NLO}}, \rho_{b}$, and $\rho_{b}^{m_{Q}}$, respectively. Thus, we have shown that the $\mu$ dependence of $m_{Q}$ is indeed canceled out.

Now we list $\rho_{a}$ and $\rho_{b}$,

$$
\begin{gathered}
\rho_{a}=q^{4}\left(\sum_{i=1}^{11} g_{1, i} G_{i}+|\theta|^{2} \sum_{i=1}^{11} g_{2, i} G_{i}\right), \\
\rho_{b}=q^{4} m_{Q} \Im \theta \sum_{i=1}^{11} g_{2, i} G_{i},
\end{gathered}
$$

where $G_{i}$ are defined as 


$$
\begin{aligned}
& G_{1}=18 G_{0,0,1}(1-v)-27 G_{0,0,2}(1-v)+3 G_{0,2,0}(1-v)-18 G_{0,2,1}(1-v)+24 G_{0,2,2}(1-v)-24 G_{2,0,0}(1-v) \\
& +18 G_{2,0,1}(1-v)-3 G_{2,0,2}(1-v)+27 G_{2,2,0}(1-v)-18 G_{2,2,1}(1-v)+12 G_{1-i \sqrt{3}, 0,0}(1-v) \\
& -18 G_{1-i \sqrt{3}, 0,1}(1-v)+15 G_{1-i \sqrt{3}, 0,2}(1-v)-15 G_{1-i \sqrt{3}, 2,0}(1-v)+18 G_{1-i \sqrt{3}, 2,1}(1-v) \\
& -12 G_{1-i \sqrt{3}, 2,2}(1-v)+12 G_{1+i \sqrt{3}, 0,0}(1-v)-18 G_{1+i \sqrt{3}, 0,1}(1-v)+15 G_{1+i \sqrt{3}, 0,2}(1-v) \\
& -15 G_{1+i \sqrt{3}, 2,0}(1-v)+18 G_{1+i \sqrt{3}, 2,1}(1-v)-12 G_{1+i \sqrt{3}, 2,2}(1-v)-3 \ln (2) G_{0,2}(1-v)+24 \ln (2) G_{2,0}(1-v) \\
& -27 \ln (2) G_{2,2}(1-v)-12 \ln (2) G_{1-i \sqrt{3}, 0}(1-v)+15 \ln (2) G_{1-i \sqrt{3}, 2}(1-v)-12 \ln (2) G_{1+i \sqrt{3}, 0}(1-v) \\
& +15 \ln (2) G_{1+i \sqrt{3}, 2}(1-v)+4\left(\pi^{2}-3 \ln (2)^{2}\right) G_{2}(1-v)-2\left(\pi^{2}-3 \ln (2)^{2}\right) G_{1-i \sqrt{3}}(1-v) \\
& -2\left(\pi^{2}-3 \ln (2)^{2}\right) G_{1+i \sqrt{3}}(1-v)+9 \zeta(3), \\
& G_{2}=4 G_{0,0,1}(1-v)-6 G_{0,0,2}(1-v)-4 G_{0,1,0}(1-v)+4 G_{0,1,2}(1-v)+6 G_{0,2,0}(1-v)-4 G_{0,2,1}(1-v) \\
& +4 G_{2,0,1}(1-v)-6 G_{2,0,2}(1-v)-4 G_{2,1,0}(1-v)+4 G_{2,1,2}(1-v)+6 G_{2,2,0}(1-v)-4 G_{2,2,1}(1-v) \\
& +4 \ln (2) G_{0,1}(1-v)-6 \ln (2) G_{0,2}(1-v)+4 \ln (2) G_{2,1}(1-v)-6 \ln (2) G_{2,2}(1-v)+3 \zeta(3), \\
& G_{3}=2 G_{0,0}(1-v)-2 G_{2,2}(1-v)-2 \ln (2) G_{0}(1-v)+\ln (2)^{2}, \\
& G_{4}=-6 G_{0,0}(1-v)+6 G_{0,2}(1-v)+6 G_{2,0}(1-v)-6 G_{2,2}(1-v)+6 \ln (2) G_{0}(1-v) \\
& -6 \ln (2) G_{2}(1-v)+\pi^{2}-3 \ln (2)^{2}, \\
& G_{5}=6 G_{0,2}(1-v)-6 G_{2,0}(1-v)+6 \ln (2) G_{2}(1-v)+\pi^{2}, \\
& G_{6}=G_{1,0}(1-v)-G_{1,2}(1-v)-\ln (2) G_{1}(1-v), \\
& G_{7}=4 G_{0,1}(1-v)-4 G_{2,1}(1-v)+\pi^{2}, \\
& G_{8}=G_{0}(1-v)+G_{2}(1-v)-\ln (2), \\
& G_{9}=G_{0}(1-v)-G_{2}(1-v)-\ln (2), \\
& G_{10}=G_{1}(1-v), \\
& G_{11}=1 .
\end{aligned}
$$

The $g_{1, i}$ are

$$
\begin{gathered}
g_{1,1}=-\frac{(v-1)^{3}(v+1)^{3}\left(3 v^{2}+5\right)}{1536 \pi^{4}}, \\
g_{1,2}=0, \\
g_{1,3}=-\frac{101 v^{12}+378 v^{10}-1149 v^{8}-5300 v^{6}+1883 v^{4}-102 v^{2}+93}{6144 \pi^{4}\left(v^{2}+3\right)^{2}}, \\
g_{1,4}=\frac{v\left(9 v^{6}-9 v^{4}+31 v^{2}-15\right)}{1152 \pi^{4}}, \\
g_{1,5}=\frac{257 v^{12}+1290 v^{10}+1119 v^{8}-500 v^{6}-1489 v^{4}+8394 v^{2}-879}{18432 \pi^{4}\left(v^{2}+3\right)^{2}},
\end{gathered}
$$




$$
\begin{gathered}
g_{1,6}=-\frac{v^{6}\left(v^{2}-3\right)}{24 \pi^{4}}, \\
g_{1,7}=-\frac{107 v^{12}+482 v^{10}+153 v^{8}-532 v^{6}+269 v^{4}+2514 v^{2}-945}{3072 \pi^{4}\left(v^{2}+3\right)^{2}}, \\
g_{1,8}=\frac{v\left(39 v^{8}+16 v^{6}+130 v^{4}+888 v^{2}-81\right)}{768 \pi^{4}\left(v^{2}+3\right)}, \\
g_{1,10}=-\frac{v\left(321 v^{8}+374 v^{6}-448 v^{4}+2874 v^{2}-945\right)}{1152 \pi^{4}\left(v^{2}+3\right)}, \\
g_{1,11}=\frac{v\left(7361 v^{6}-12289 v^{4}+18199 v^{2}-9863\right)}{18432 \pi^{4}} .2855 v^{8}-39174 v^{6}-8018 v^{4}+6397 v^{2}-14469 \\
\left.36864 \pi^{4}+3\right)
\end{gathered}
$$

The $g_{2, i}$ are

$$
\begin{aligned}
& g_{2,1}=-\frac{(v-1)^{2}(v+1)^{2}\left(v^{2}-5\right)\left(v^{2}+3\right)}{256 \pi^{4}}, \\
& g_{2,2}=\frac{(v-1)^{2}(v+1)^{2}}{8 \pi^{4}} \\
& g_{2,3}=-\frac{11 v^{10}+133 v^{8}-4482 v^{6}-2054 v^{4}+3911 v^{2}-591}{3072 \pi^{4}\left(v^{2}+3\right)}, \\
& g_{2,4}=\frac{v\left(3 v^{6}-11 v^{4}+69 v^{2}-45\right)}{192 \pi^{4}}, \\
& g_{2,5}=\frac{347 v^{10}-419 v^{8}-2610 v^{6}+154 v^{4}+21287 v^{2}-12615}{9216 \pi^{4}\left(v^{2}+3\right)}, \\
& g_{2,6}=-\frac{v^{2}\left(v^{2}+2\right)\left(v^{4}-6 v^{2}+3\right)}{12 \pi^{4}}, \\
& g_{2,7}=-\frac{137 v^{10}-173 v^{8}-894 v^{6}+782 v^{4}+5605 v^{2}-3921}{1536 \pi^{4}\left(v^{2}+3\right)}, \\
& g_{2,8}=\frac{v\left(42 v^{6}-235 v^{4}+1090 v^{2}-501\right)}{192 \pi^{4}}, \\
& g_{2,9}=\frac{4823 v^{8}-19532 v^{6}-40278 v^{4}+26932 v^{2}-9961}{18432 \pi^{4}} \\
& g_{2,10}=-\frac{v\left(411 v^{6}-1831 v^{4}+7069 v^{2}-3921\right)}{576 \pi^{4}}, \\
& g_{2,11}=\frac{v\left(8111 v^{6}-29663 v^{4}+104473 v^{2}-72697\right)}{9216 \pi^{4}} .
\end{aligned}
$$


And finally, the $g_{3, i}$ are

$$
\begin{aligned}
& g_{3,1}=-\frac{(v-1)(v+1)\left(v^{4}+2 v^{2}+5\right)}{32 \pi^{4}}, \\
& g_{3,2}=-\frac{3(v-1)(v+1)}{4 \pi^{4}}, \\
& g_{3,3}=-\frac{8 v^{10}+35 v^{8}+20 v^{6}+114 v^{4}+556 v^{2}-221}{32 \pi^{4}\left(v^{2}+3\right)^{2}}, \\
& g_{3,4}=\frac{v\left(v^{2}+3\right)\left(3 v^{2}-5\right)}{24 \pi^{4}}, \\
& g_{3,5}=\frac{20 v^{10}+155 v^{8}+500 v^{6}+666 v^{4}-704 v^{2}-1661}{96 \pi^{4}\left(v^{2}+3\right)^{2}}, \\
& g_{3,6}=-\frac{v^{2}\left(v^{4}-3 v^{2}+6\right)}{2 \pi^{4}}, \\
& g_{3,7}=-\frac{2 v^{10}+14 v^{8}+35 v^{6}+29 v^{4}-57 v^{2}-87}{4 \pi^{4}\left(v^{2}+3\right)^{2}}, \\
& g_{3,8}=\frac{v\left(3 v^{6}+13 v^{4}-24 v^{2}-120\right)}{4 \pi^{4}\left(v^{2}+3\right)}, \\
& g_{3,9}=\frac{89 v^{8}+177 v^{6}-39 v^{4}+859 v^{2}+450}{48 \pi^{4}\left(v^{2}+3\right)}, \\
& g_{3,10}=-\frac{v\left(12 v^{6}+43 v^{4}-33 v^{2}-174\right)}{3 \pi^{4}\left(v^{2}+3\right)},
\end{aligned}
$$

$$
\begin{aligned}
& \left.\rho_{1,1}^{\mathrm{NLO}}\right|_{m_{Q}^{\text {on-shell }}, q^{2} \rightarrow 4 m_{Q}^{2}} \\
& =\frac{\alpha_{s}}{2 \pi}\left(4 m_{Q}^{2}\right)^{2}\left[\frac{1}{32 \pi^{2}}\left(1+4|\theta|^{2}\right) v^{6}+\frac{2\left(1161+70 \pi^{2}-945 \ln 2-630 \ln v\right)+315 \ln \left(\frac{\mu^{2}}{m_{Q}^{2}}\right)}{7350 \pi^{4}}\left(1+4|\theta|^{2}\right) v^{7}+\mathcal{O}\left(v^{8}\right)\right],
\end{aligned}
$$

$$
\left.\rho_{2,1}^{\mathrm{NLO}}\right|_{m_{Q}^{\text {on-shell }, q^{2} \rightarrow 4 m_{Q}^{2}}}=\frac{\alpha_{s}}{2 \pi}\left(4 m_{Q}^{2}\right)^{2} m_{Q} \Im \theta\left[-\frac{1}{4 \pi^{2}} v^{6}-\frac{4\left(2\left(1161+70 \pi^{2}-945 \ln 2-630 \ln v\right)+315 \ln \left(\frac{\mu^{2}}{m_{Q}^{2}}\right)\right)}{3675 \pi^{4}} v^{7}+\mathcal{O}\left(v^{8}\right)\right] \text {, }
$$

$$
\left.\rho_{1,1}^{\mathrm{NLO}}\right|_{m_{Q}^{\overline{\mathrm{MS}}}, q^{2} \rightarrow 4 m_{Q}^{2}}=\left.\rho_{1,1}^{\mathrm{NLO}}\right|_{m_{Q}^{\text {on-shell }}, q^{2} \rightarrow 4 m_{Q}^{2}}+\frac{\alpha_{s}}{2 \pi}\left[\frac{8}{3}+2 \ln \left(\frac{\mu^{2}}{m_{Q}^{2}}\right)\right]\left(4 m_{Q}^{2}\right)^{2}\left[-\frac{3}{20 \pi^{4}}\left(1+4|\theta|^{2}\right) v^{5}-\frac{3}{140 \pi^{4}}\left(5+28|\theta|^{2}\right) v^{7}+\mathcal{O}\left(v^{8}\right)\right],
$$




$$
\left.\rho_{2,1}^{\mathrm{NLO}}\right|_{m_{Q}^{\overline{\mathrm{MS}}}, q^{2} \rightarrow 4 m_{Q}^{2}}=\left.\rho_{2,1}^{\mathrm{NLO}}\right|_{m_{Q}^{\text {on-shell }}, q^{2} \rightarrow 4 m_{Q}^{2}}+\frac{\alpha_{s}}{2 \pi}\left[\frac{8}{3}+2 \ln \left(\frac{\mu^{2}}{m_{Q}^{2}}\right)\right]\left(4 m_{Q}^{2}\right)^{2} m_{Q} \Im \theta\left[\frac{6}{5 \pi^{4}} v^{5}+\frac{54}{35 \pi^{4}} v^{7}+\mathcal{O}\left(v^{8}\right)\right] .
$$

The $v^{6}$ terms in the above expressions correspond to the Coulombic singularities generated by the gluon exchange between two heavy quarks. The $v^{5}$ terms in Eqs. (A71) and (A72) come from the renormalization-scheme difference of $m_{Q}$, i.e., Eqs. (A10) and (A11).

We also present our NLO result before renormalization in terms of the coefficients of master integrals,

$$
C_{1}^{\mathrm{NLO}}=i \pi\left(\frac{\mu^{2}}{q^{2}}\right)^{3 \varepsilon} \sum_{k}\left(\alpha_{k} \not 1+\beta_{k}\right) I_{k}
$$

where $\alpha_{k}$ and $\beta_{k}$ are real. Thus, by the definition (5) we have

$$
\begin{aligned}
& \rho_{1,1}^{\mathrm{NLO}}=\left(\frac{\mu^{2}}{q^{2}}\right)^{3 \varepsilon} \sum_{k} \alpha_{k} \Re I_{k}, \\
& \rho_{2,1}^{\mathrm{NLO}}=\left(\frac{\mu^{2}}{q^{2}}\right)^{3 \varepsilon} \sum_{k} \beta_{k} \Re I_{k},
\end{aligned}
$$

where the master integrals $I_{k}$ are defined to be dimensionless, which are the same as those in Eq. (15). Note that the 29 master integrals in Eq. (15) contain some symmetries, that is, some of them can be related to each other by shifting loop momenta. After using these symmetries, we are only left with 14 master integrals, which are defined as

$$
\begin{gathered}
I_{1}=\left(q^{2}\right)^{3 \varepsilon-3} \prod_{i=1}^{3} \int \frac{\mathrm{d}^{d} l_{i}}{(2 \pi)^{d}} \frac{1}{l_{i}^{2}-m_{Q}^{2}}, \\
I_{2}=\left(q^{2}\right)^{3 \varepsilon-2} \int\left(\prod_{i=1}^{3} \frac{\mathrm{d}^{d} l_{i}}{(2 \pi)^{d}}\right) \frac{1}{\left(l_{1}+l_{2}+l_{3}\right)^{2} l_{1}^{2}\left[l_{2}^{2}-m_{Q}^{2}\right]\left[l_{3}^{2}-m_{Q}^{2}\right]}, \\
I_{3}=\left(q^{2}\right)^{3 \varepsilon-2} \int\left(\prod_{i=1}^{3} \frac{\mathrm{d}^{d} l_{i}}{(2 \pi)^{d}}\right) \frac{1}{\left[\left(l_{1}+l_{2}+l_{3}\right)^{2}-m_{Q}^{2}\right]\left[l_{1}^{2}-m_{Q}^{2}\right]\left[l_{2}^{2}-m_{Q}^{2}\right]\left[l_{3}^{2}-m_{Q}^{2}\right]} \\
I_{4}=\left(q^{2}\right)^{3 \varepsilon-2} \int\left(\prod_{i=1}^{3} \frac{\mathrm{d}^{d} l_{i}}{(2 \pi)^{d}}\right) \frac{1}{\left(q-l_{1}-l_{2}-l_{3}\right)^{2} l_{1}^{2}\left[l_{2}^{2}-m_{Q}^{2}\right]\left[l_{3}^{2}-m_{Q}^{2}\right]} \\
I_{6}=\left(q^{2}\right)^{3 \varepsilon-1} \int\left(\prod_{i=1}^{3 \varepsilon-1} \frac{\mathrm{d}^{d} l_{i}}{(2 \pi)^{d}}\right) \frac{1}{\left(q-l_{1}-l_{2}-l_{3}\right)^{2} l_{1}^{2}\left[l_{2}^{2}-m_{Q}^{2}\right]\left[l_{3}^{2}-m_{Q}^{2}\right]^{2}}, \\
I_{7} l_{i} \\
I_{7}=\left(q^{2}\right)^{3 \varepsilon-2} \int\left(\prod_{i=1}^{2} \frac{\mathrm{d}^{d} l_{i}}{(2 \pi)^{d}}\right) \frac{1}{\left(q-l_{1}-l_{2}-l_{3}\right)^{4} l_{1}^{2}\left[l_{2}^{2}-m_{Q}^{2}\right]\left[l_{3}^{2}-m_{Q}^{2}\right]}, \\
I_{8}=\left(q^{2}\right)^{3 \varepsilon-1} \int\left(\prod_{i=1}^{2} \frac{\mathrm{d}^{d} l_{i}}{(2 \pi)^{d}}\right) \frac{\mathrm{d}^{d} l_{3}}{\left(q-l_{1}-l_{2}\right)^{2}\left[l_{1}^{2}-m_{Q}^{2}\right]\left[l_{2}^{2}-m_{Q}^{2}\right]} \frac{1}{(2 \pi)^{d}} \frac{1}{l_{3}^{2}-m_{Q}^{2}} \\
\left.(2 \pi)^{d}\right) \frac{\mathrm{d}^{d} l_{3}}{\left(q-l_{2}\right)^{4}\left[l_{1}^{2}-m_{Q}^{2}\right]\left[l_{2}^{2}-m_{Q}^{2}\right]} \int \frac{1}{(2 \pi)^{d}} \frac{\mathrm{d}^{d} l_{3}^{2}}{l_{3}^{2}-m_{Q}^{2}}
\end{gathered}
$$




$$
\begin{gathered}
I_{10}=\left(q^{2}\right)^{3 \varepsilon-1} \int\left(\prod_{i=1}^{3} \frac{\mathrm{d}^{d} l_{i}}{(2 \pi)^{d}}\right) \frac{1}{\left(q-l_{1}\right)^{2}\left[\left(l_{1}-l_{2}\right)^{2}-m_{Q}^{2}\right]\left[l_{2}^{2}-m_{Q}^{2}\right]\left[\left(l_{1}-l_{3}\right)^{2}-m_{Q}^{2}\right]\left[l_{3}^{2}-m_{Q}^{2}\right]}, \\
I_{11}=\left(q^{2}\right)^{3 \varepsilon} \int\left(\prod_{i=1}^{3} \frac{\mathrm{d}^{d} l_{i}}{(2 \pi)^{d}}\right) \frac{1}{\left(q-l_{1}\right)^{4}\left[\left(l_{1}-l_{2}\right)^{2}-m_{Q}^{2}\right]\left[l_{2}^{2}-m_{Q}^{2}\right]\left[\left(l_{1}-l_{3}\right)^{2}-m_{Q}^{2}\right]\left[l_{3}^{2}-m_{Q}^{2}\right]} \\
I_{12}=\left(q^{2}\right)^{3 \varepsilon-1} \int\left(\prod_{i=1}^{3} \frac{\mathrm{d}^{d} l_{i}}{(2 \pi)^{d}}\right) \frac{1}{\left[\left(q-l_{1}\right)^{2}-m_{Q}^{2}\right]\left(l_{1}-l_{2}\right)^{2}\left[l_{2}^{2}-m_{Q}^{2}\right]\left(l_{1}-l_{3}\right)^{2}\left[l_{3}^{2}-m_{Q}^{2}\right]} \\
I_{13}=\left(q^{2}\right)^{3 \varepsilon} \int\left(\prod_{i=1}^{3} \frac{\mathrm{d}^{d} l_{i}}{(2 \pi)^{d}}\right) \frac{1}{\left[\left(q-l_{1}\right)^{2}-m_{Q}^{2}\right]^{2}\left(l_{1}-l_{2}\right)^{2}\left[l_{2}^{2}-m_{Q}^{2}\right]\left(l_{1}-l_{3}\right)^{2}\left[l_{3}^{2}-m_{Q}^{2}\right]} \\
I_{14}=\left(q^{2}\right)^{3 \varepsilon} \int\left(\prod_{i=1}^{3} \frac{\mathrm{d}^{d} l_{i}}{(2 \pi)^{d}}\right) \frac{1}{\left[\left(q-l_{1}\right)^{2}-m_{Q}^{2}\right]\left(l_{1}-l_{2}\right)^{2}\left[l_{2}^{2}-m_{Q}^{2}\right]\left(l_{1}-l_{3}\right)^{2}\left[l_{3}^{2}-m_{Q}^{2}\right]^{2}}
\end{gathered}
$$

Using the differential equation method, we obtain the real part of the master integrals up to $\mathcal{O}\left(\varepsilon^{2}\right)$ in terms of Goncharov polylogarithms. The explicit expressions of $\alpha_{i}$, $\beta_{i}$, and $\Re I_{i}$ are lengthy and will be presented in the ancillary file of the arXiv preprint.

\section{APPENDIX B: HIGHER-DIMENSIONAL OPERATORS}

In addition to $\langle\bar{q} q\rangle$ and $\left\langle g_{s}^{2} G G\right\rangle$ operators, we also calculate the Wilson coefficients of the $\left\langle g_{s} \bar{q} q G\right\rangle$ operator up to the leading contributions,

$$
\begin{aligned}
& \rho_{1,\left\langle g_{s} \bar{q} q G\right\rangle}^{\mathrm{LO}}=\rho_{c}^{\left\langle g_{s} \bar{q} q G\right\rangle}+\rho_{c}^{\langle\bar{q} q\rangle}, \\
& \rho_{2,\left\langle g_{s} \bar{q} q G\right\rangle}^{\mathrm{LO}}=\rho_{d}^{\left\langle g_{s} \bar{q} q G\right\rangle}+\rho_{d}^{\langle\bar{q} q\rangle},
\end{aligned}
$$

where $\rho_{c}^{\left\langle g_{s} \bar{q} q G\right\rangle}$ and $\rho_{d}^{\left\langle g_{s} \bar{q} q G\right\rangle}$ come directly from the $\left\langle g_{s} \bar{q} q G\right\rangle$ operator, while $\rho_{c}^{\langle\bar{q} q\rangle}$ and $\rho_{d}^{\langle\bar{q} q\rangle}$ are contributions from the expansion of the $\langle\bar{q} q\rangle$ operator [37]. Here $\rho_{c}^{\left\langle g_{s} \bar{q} q G\right\rangle}$ and $\rho_{d}^{\left\langle g_{s} \bar{q} q G\right\rangle}$ are

$$
\begin{gathered}
\rho_{c}^{\left\langle g_{s} \bar{q} q G\right\rangle}=\frac{1}{8 \pi^{2}} \frac{m_{Q}}{q^{2}} \Im \theta \frac{5 v^{2}+7}{v}, \\
\rho_{d}^{\left\langle g_{s} \bar{q} q G\right\rangle}=-\frac{1}{32 \pi^{2}} \frac{v^{2}+3}{v}+\frac{1}{2 \pi^{2}}|\theta|^{2} \frac{v^{2}-1}{v},
\end{gathered}
$$

and $\rho_{c}^{\langle\bar{q} q\rangle}$ and $\rho_{d}^{\langle\bar{q} q\rangle}$ are

$$
\begin{gathered}
\rho_{c}^{\langle\bar{q} q\rangle}=\frac{3}{16 \pi^{2}} \frac{m_{Q}}{q^{2}} \Im \theta \frac{\left(v^{2}-1\right)\left(3 v^{2}-1\right)}{v^{3}}, \quad \text { (B5) } \\
\rho_{d}^{\langle\bar{q} q\rangle}=\frac{1}{128 \pi^{2}} \frac{v^{6}+3 v^{4}+15 v^{2}-3}{v^{3}}+\frac{3}{32 \pi^{2}}|\theta|^{2} \frac{\left(v^{2}-1\right)^{3}}{v^{3}} .
\end{gathered}
$$

Again, with the help of Eq. (14), our result confirms previous calculations [16].

Note that $\rho_{c}^{\langle\bar{q} q\rangle}$ and $\rho_{d}^{\langle\bar{q} q\rangle}$ contain Coulombic-like singularities, which will cause the integral over $s$ in Eq. (7) to diverge at the threshold. Thus, we cannot use the above results in our sum rule analysis directly. To deal with these singularities, we may consider resumming the leading Coulombic interaction $\left(\frac{\alpha_{s}}{v}\right)^{n}$ between two heavy quarks $Q$. The amplitude of the $\left(Q^{a} C \Gamma_{1} Q^{b}\right)$ part of the baryon current is multiplied by the Sommerfeld factor $[17,57,58]$,

$$
S(v)=\frac{\frac{C \pi \alpha_{s}}{v}}{1-\exp \left(-\frac{C \pi \alpha_{s}}{v}\right)},
$$

where $C$ is the color factor. In our case, $Q^{a} Q^{b}$ forms a color antitriplet, so $C=\frac{2}{3}$. The resummed $\rho_{c}^{\langle\bar{q} q\rangle}$ and $\rho_{d}^{\langle\bar{q} q\rangle}$ are

$$
\begin{aligned}
\rho_{c}^{\langle\bar{q} q\rangle}= & \frac{3}{16 \pi^{2}} \frac{m_{Q}}{q^{2}} \Im \theta \frac{\left(v^{2}-1\right)}{v^{3}}\left[S(v)\left(3 v^{2}-1\right)\right. \\
& \left.+S^{\prime}(v) v\left(v^{2}+1\right)-S^{\prime \prime}(v) v^{2}\left(v^{2}-1\right)\right], \quad \text { (B8) } \\
\rho_{d}^{\langle\bar{q} q\rangle}= & \frac{1}{128 \pi^{2}} \frac{1}{v^{3}}\left[S(v)\left(v^{6}+3 v^{4}+15 v^{2}-3\right)\right. \\
& \left.-S^{\prime}(v) v\left(v^{4}-1\right)\left(v^{2}+3\right)-S^{\prime \prime}(v) v^{2}\left(v^{2}-1\right)^{2}\left(v^{2}-3\right)\right] \\
& +\frac{3}{32 \pi^{2}}|\theta|^{2} \frac{\left(v^{2}-1\right)^{3}}{v^{3}}\left[S(v)-S^{\prime}(v) v-S^{\prime \prime}(v) v^{2}\right],
\end{aligned}
$$

where the prime denotes the derivative with respect to $v$. After resummation, the Coulombic-like singularities are regularized by the Sommerfeld factor, and the integral over $s$ in Eq. (7) converges.

Now we can include the $\left\langle g_{s} \bar{q} q G\right\rangle$ condensate in our sum rule analysis, and investigate its contribution to the sum rule 
and $m_{\Xi_{c c}^{++}}$estimation. The vacuum condensate parameter is taken to be $[16-21,41]$

$$
\left\langle g_{s} \bar{q} q G\right\rangle(2 \mathrm{GeV})=\left(0.8 \pm 0.2 \mathrm{GeV}^{2}\right) \times\langle\bar{q} q\rangle(2 \mathrm{GeV})
$$

The vacuum condensate can be evolved according to its one-loop anomalous dimensions: $\gamma_{\left\langle g_{s} \bar{q} q G\right\rangle}=-\frac{\gamma_{m_{q}}}{6}$ [55]. The relative importance of each condensate term in the OPE, including $\left\langle g_{s} \bar{q} q G\right\rangle$, is shown in Fig. 6.

By defining the condensate term of $O_{i}$ to be

$$
c_{i}=\left\langle O_{i}\right\rangle \int_{s_{\mathrm{th}}}^{\infty} \mathrm{d} s \rho_{1, i}(s) e^{-\frac{s}{m_{B}^{2}}}
$$

the ratios between consecutive terms in the $m_{Q}^{\overline{\mathrm{MS}}}$ scheme at central values of all parameters are

$$
\left|\frac{c_{g_{s}^{2} G G}}{c_{\bar{q} q}}\right|=24 \%, \quad\left|\frac{c_{g_{s} \bar{q} q G}}{c_{g_{s}^{2} G G}}\right|=8 \% .
$$

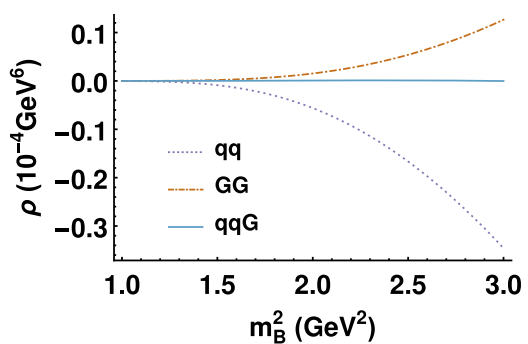

FIG. 6. Contributions of various terms on the right-hand side of Eq. (7).

The $c_{g_{s} \bar{q} q G}$ contribution to the right-hand side of Eq. (7) is less than $0.6 \%$, and the estimated $m_{\Xi_{c c}^{++}}$changes by less than $0.3 \%$ in both the LO and LO + NLO cases. We see that the OPE seems to show good convergence, and it might be a good approximation to neglect the contributions of operators with dimension larger than four in the sum rule (7). Nevertheless, it is certainly helpful to have a systematic study for the contributions of higher-dimensional operators in the future.
[1] R. Aaij et al. (LHCb Collaboration), Phys. Rev. Lett. 119, 112001 (2017).

[2] M. Mattson et al. (SELEX Collaboration), Phys. Rev. Lett. 89, 112001 (2002).

[3] B. Aubert et al. (BABAR Collaboration), Phys. Rev. D 74, 011103 (2006).

[4] R. Chistov et al. (Belle Collaboration), Phys. Rev. Lett. 97, 162001 (2006).

[5] R. J. Hudspith, A. Francis, R. Lewis, and K. Maltman, Proc.Sci. LATTICE2016 (2017) 133.

[6] Y. Namekawa et al. (PACS-CS Collaboration), Phys. Rev. D 87, 094512 (2013).

[7] R. Lewis, N. Mathur, and R. M. Woloshyn, Phys. Rev. D 64, 094509 (2001).

[8] Z.-F. Sun and M. J. V. Vacas, Phys. Rev. D 93, 094002 (2016).

[9] A. V. Kiselev, A. V. Berezhnoy, and A. K. Likhoded, Yad. Fiz. 81, 356 (2018) [Phys. At. Nucl. 81, 369 (2018)].

[10] Z. Shah and A. K. Rai, Eur. Phys. J. C 77, 129 (2017).

[11] A. N. Gadaria, N. R. Soni, and J. N. Pandya, DAE Symp. Nucl. Phys. 61, 698 (2016).

[12] W. Roberts and M. Pervin, Int. J. Mod. Phys. A 23, 2817 (2008).

[13] D. Ebert, R. N. Faustov, V. O. Galkin, and A. P. Martynenko, Phys. Rev. D 66, 014008 (2002).

[14] M. A. Shifman, A. I. Vainshtein, and V. I. Zakharov, Nucl. Phys. B147, 385 (1979).

[15] M. A. Shifman, A. I. Vainshtein, and V. I. Zakharov, Nucl. Phys. B147, 448 (1979).

[16] E. Bagan, M. Chabab, and S. Narison, Phys. Lett. B 306, 350 (1993).
[17] V. V. Kiselev and A. I. Onishchenko, Nucl. Phys. B581, 432 (2000).

[18] J.-R. Zhang and M.-Q. Huang, Phys. Rev. D 78, 094007 (2008).

[19] Z.-G. Wang, Eur. Phys. J. A 45, 267 (2010).

[20] L. Tang, X.-H. Yuan, C.-F. Qiao, and X.-Q. Li, Commun. Theor. Phys. 57, 435 (2012).

[21] T. M. Aliev, K. Azizi, and M. Savci, Nucl. Phys. A895, 59 (2012).

[22] H.-X. Chen, Q. Mao, W. Chen, X. Liu, and S.-L. Zhu, Phys. Rev. D 96, 031501 (2017); 96, 119902(E) (2017).

[23] L. J. Reinders, H. Rubinstein, and S. Yazaki, Phys. Rep. 127, 1 (1985).

[24] M. Jamin, Z. Phys. C 37, 635 (1988).

[25] A. A. Ovchinnikov, A. A. Pivovarov, and L. R. Surguladze, Int. J. Mod. Phys. A 06, 2025 (1991).

[26] J. S. Schwinger, Particles, Sources, and Fields (Perseus books, Reading, Massachusetts, 1998), Vol. 3.

[27] A. Maier and P. Marquard, Nucl. Phys. B859, 1 (2012).

[28] P. A. Baikov, K. G. Chetyrkin, and J. H. Kuhn, Nucl. Phys. B, Proc. Suppl. 189, 49 (2009).

[29] K. G. Chetyrkin, R. V. Harlander, and J. H. Kuhn, Nucl. Phys. B586, 56 (2000); B634, 413(E) (2002).

[30] P. A. Baikov, K. G. Chetyrkin, and J. H. Kuhn, Phys. Rev. Lett. 101, 012002 (2008).

[31] P. A. Baikov, K. G. Chetyrkin, and J. H. Kuhn, Nucl. Phys. B, Proc. Suppl. 135, 243 (2004).

[32] S. Groote, J. G. Korner, and A. A. Pivovarov, Eur. Phys. J. C 58, 355 (2008). 
[33] K. G. Chetyrkin and F. V. Tkachov, Nucl. Phys. B192, 159 (1981).

[34] S. Laporta, Int. J. Mod. Phys. A 15, 5087 (2000).

[35] J. M. Henn, Phys. Rev. Lett. 110, 251601 (2013).

[36] J. M. Henn, J. Phys. A 48, 153001 (2015).

[37] B. L. Ioffe, Nucl. Phys. B188, 317 (1981); B191, 591(E) (1981).

[38] B. L. Ioffe, Z. Phys. C 18, 67 (1983).

[39] Y. Chung, H. G. Dosch, M. Kremer, and D. Schall, Nucl. Phys. B197, 55 (1982).

[40] D. B. Leinweber, Ann. Phys. (N.Y.) 254, 328 (1997).

[41] S. Narison and R. Albuquerque, Phys. Lett. B 694, 217 (2011).

[42] J. Kublbeck, M. Bohm, and A. Denner, Comput. Phys. Commun. 60, 165 (1990).

[43] T. Hahn, Comput. Phys. Commun. 140, 418 (2001).

[44] R. Mertig, M. Bohm, and A. Denner, Comput. Phys. Commun. 64, 345 (1991).

[45] V. Shtabovenko, R. Mertig, and F. Orellana, Comput. Phys. Commun. 207, 432 (2016).
[46] A. V. Smirnov, Comput. Phys. Commun. 189, 182 (2015).

[47] R. N. Lee, J. Phys. Conf. Ser. 523, 012059 (2014).

[48] R. N. Lee, J. High Energy Phys. 04 (2015) 108.

[49] A. B. Goncharov, arXiv:math/0103059.

[50] M. E. Peskin, Phys. Lett. 88B, 128 (1979).

[51] C. Patrignani et al. (Particle Data Group), Chin. Phys. C 40, 100001 (2016).

[52] C. A. Dominguez, G. R. Gluckman, and N. Paver, Phys. Lett. B 333, 184 (1994).

[53] C. A. Dominguez, L. A. Hernandez, and K. Schilcher, J. High Energy Phys. 07 (2015) 110.

[54] S. Aoki et al., Eur. Phys. J. C 77, 112 (2017).

[55] R. M. Albuquerque, Charmonium exotic states, Ph.D. thesis, Sao Paulo U., 2013.

[56] R. A. Bertlmann, Nucl. Phys. B204, 387 (1982).

[57] A. Sommerfeld, Atombau und Spektrallinien (Friedr. Vieweg \& Sohn, Braunschweig, 1939).

[58] J. Portoles and P. D. Ruiz-Femenia, Eur. Phys. J. C 24, 439 (2002). 